\title{
Research Update on Modern Medicine and Surgery
}

\section{Simon Raymond*}

Alumnus Melbourne University, Parkville, Victoria, Australia

\author{
Abstract \\ The following topics represent perhaps the most current and relevant areas of medicine, law and society: \\ Medicine: Developing and Enhancing Medicine and Surgery \\ -Application of quantum physics (advanced physics) to medicine \& surgery \\ Law: IP related offences \\ Society: Infectious diseases and protection type issues
}

\section{Application of quantum physics to medicine and surgery}

The current author is the researcher who has conceptualised the new pathway forward for medicine and surgery which is that involving the "Application of Quantum Physics to Medicine and Surgery" [1-9].

This is essentially the application of advanced physics to medicine and surgery. Prior to this, medicine and surgery has had basic science teaching in physics that is combined with biology to form a merged teaching unit termed 'physiology' (physics and biology, as a merged unit).

The basic level physics included in the physiology (and other teachings) is more along the lines of that found in classical physics.

In brief, the main difference between quantum and classical physics:

1. Classical physics focuses more on a paradigm or basis for understanding in which orderly, sequential (or, otherwise easy to follow step by step in manner) explanations occur for describing relationships between events (and describing relationships between variables of interest in general).

2. Quantum physics focuses on a more in depth understanding of events that involves relationships between variables and events that are not hindered by any restrictive preconceptions There is no requirement for a time variable, or other variable, of general reference. Events and other variable relationships are therefore simultaneously occurring. Core concepts include the duality of light and that it acts in both particle and wave form (by way of probability distribution), and the similarity to this (wave distribution) for matter, commencing with electrons. It is also of note that awareness and consciousness would seem notable, as indicated by concepts including observer effect and experiments including the double slit experiment.

Notable quantum physicists include: Niels Bohr, Werner Heisenberg, Erwin Schröndinger, Albert Einstein (theoretical physicist), Pascual Jordan, Anton Zeilinger, Julian Schwinger.

Examples surrounding optics, neurology and related areas seem a good starting point to highlight the difference between what is presently in medicine and surgery and the differences with quantum physics in addition to the benefit and reasons for the updating of the profession. This is based on the connection to light, electrical activity and awareness.

\section{For instance:}

1. An understanding that the central beam theory (pinhole aperture test) may perhaps be better explained by way of scientific principles, in quantum physics, revolving around light acting in both wave and particle forms and, by application of the pinhole aperture, light may arguably as a result hit the retina more predominantly in particle form, and subsequently in a more concentrated manner, thereby increasing visual acuity [1-9].

2. (2) Monocular abilities to judge depth (depth perception) may perhaps be better explained through interaction of diffraction wave patterns (Example, from points of different distance relationships) with resulting superposition (constructive interference) and destructive interference, and accompanying peaks and troughs, with neurological calculation of time and distance relationships based on related analyses of the interference as described, as opposed to historical explanations such as texture gradient, interposition, relative size Etc. Interestingly, partial coherence interferometry (used in ophthalmology for measurement of ocular axial length in calculation regarding the IOL to be implanted in the surgical eye), utilises such principles. The author intends to seek patent rights regarding application of quantum physics to medicine and surgery [1-9].

NB: Historical explanations may still hold some practical relevance

\section{In summary}

The current author is the researcher who has conceptualised the new pathway forward for medicine and surgery which is that involving the "Application of Quantum Physics to Medicine and Surgery". Broad description using multiple examples was first provided with regards to how this adds improved understanding to medicine and surgery, followed by specific examples with regards to this.

\section{Site attachment inhibition}

In previous conferences and publications, it has been detailed that the current author and researcher has conceptualised and developed the new, or third, branch of antimicrobial therapeutics, namely site

*Corresponding author: Raymond S, Alumnus Melbourne University, Parkville Victoria, Australia, Tel: +61 38344 1746; E-mail: simonraymondcontact@gmail.com

Received July 07, 2018; Accepted July 25, 2018; Published August 05, 2018

Citation: Raymond S (2018) Research Update on Modern Medicine and Surgery. Mol Biol 7: 216. doi: 10.4172/2168-9547.1000216

Copyright: () 2018 Raymond S. This is an open-access article distributed under the terms of the Creative Commons Attribution License, which permits unrestricted use, distribution, and reproduction in any medium, provided the original author and source are credited. 
attachment inhibition which involves negation of cellular attachment to (or, negation of entry and transfer into) the human cellular biology by infective agents. This is based on the issues with metaphorical superbugs, development of antimicrobial resistance, and the general lack of success currently with respect to the previous two branches which have focused on:

1. Replication of infective agent and,

2. Immune system enhancement.

Recent talks have highlighted that site attachment inhibition is intended to consist of both:

(A) Treatment of established infections,

(B) New generation immunization programs (preventative treatment).

New generation immunization programs, based on prenatal stem cell therapy in the prenatal period and earlier spanning back to spermatogenesis and oogenesis, is intended to involve gene mutagenesis, and knockout. New content presented in recent talks involves methods for dealing with association and causation issues.These methods include use of technologies including CRISPR and CRISPR-Cas9 [5-9].

\section{Infectious disease and global society}

Respect for biology by ethics committees and community members has been detailed in previous publications.

Supporting that awareness of infective agents should be taken seriously:

1. There is consideration by respected universities regarding awareness of computers and the need to consider whether computers should be provided similar rights to that of human rights $[11,12]$.

2. There is merger occurring with the IT industry. Examples include three-dimensional printing of biology [8].

3. There is support for the opinion that infective agents may contain awareness and this is detailed in previous publications. It is supported further in this publication by the ability of infective agents to sense surroundings in the contexts of discriminating between self and foreign [8].

Furthermore, previous publications by the current author have explained that such infective agents could have gained such abilities through mimicry of the neurological system. This is discussed in a reference further [5].

Following on from the above, it is interesting to analyse the current period which is termed by some as world war type climate and it is interesting to discuss perhaps whether there is any relationship between war and medicine based on examples including: USA has had roles in areas of war including that in Israel and such regions and also in regions containing infectious disease issues including Africa [8]. The use of words which relate to infectious disease almost in Israel in use with regards to macro beings during historical world wars and in Africa more use of infectious disease terminology with regards to microorganisms.

This may be discussed in future topics. At this stage, the views of the author with regards to what may perhaps assist with developing guidance for doctors (e.g. clinical doctors) on such topics is presented in previous publications including discrimination between infective agents and persons, for instance microorganism compared with macro or human (physical) being.
The above however may need to be revised with the findings that microorganisms may contain awareness.

\section{Copying neurology and other modern intellectual property offences}

Intellectual Property (IP) has become one of the most important resources in modern society, and IP theft is becoming recognised as one of the most important of modern offences.

There will be discussion in future publications regarding whether certain issues comprise modern offences, including that with regards to copying of neurology (in situations which are not proper), in addition to surrounding topics $[5-8,11,12]$.

\section{Summary of report}

The content of this manuscript has been taught in lectures by the current author Dr Simon Raymond at the following conference and congress proceedings:

1. Introduction to "Application of Quantum Physics to Medicine and Surgery" and "Site Attachment Inhibition"

-Annual Conference on Microbial Pathogenesis, Infectious Disease, Antimicrobials and Drug Resistance Aug 23-24 2017, Toronto, Canada.

-World Congress \& Expo on Biotechnology and Bioengineering March 27-29 2017, Dubai, UAE.

-6th International Conference on Immunology October 24-26 2016, Chicago, USA.

2. Dealing with Association and Causation Issues in Site Attachment Inhibition

-Proceedings of Global Summit on Immunology and Cell Biology \& Global Congress on Bacteriology and Infectious Diseases June 25-26 2018, Amsterdam, Netherlands.

-9th European Immunology Conference Associated with Antibody Engineering June 14-16 2018, Rome, Italy.

3. New Generation Immunization Schedules for Site Attachment Inhibition

-2nd World Vaccines and Immunology Congress October 15-16 2018, Tokyo, Japan

\section{Ethical Considerations Part 1}

-Global Experts Meeting on Infectious Diseases September 03-04 2018, Tokyo, Japan.

The content of this manuscript comprises what are perhaps the most modern areas of medicine, law and society:

\section{Medicine}

Advancing Medicine and Surgery

1. The application of quantum physics (or, advanced physics) to medicine and surgery to enhance the practice

2. Site attachment inhibition to be explored as a way to deal with one of the most notable current medical topics (infectious disease).

Law

1. IP offences are becoming recognised as one of the most important modern offences 
2. IP offences relating to medicine and surgery may be relevant including copying of neurology.

\section{Society}

1. The impact of infectious disease on society

2. The connection between war and medicine and issues such as macro being and microorganism distinction perhaps not being enough given the content such as that presented.

Conference presentations have occurred globally including Spain, USA, Japan, Czech Republic, UK, China, Australia, Dubai, Netherlands, Switzerland.

Future presentations will cover the above topics and related areas.

\section{References}

1. Raymond S. (2016) Development of New Strategic Pathways for Antiviral Therapy. J Clin Cell Immunol 7: 5.

2. Raymond S. (2016) Consciousness and the Development of New Strategic Pathways for Antiviral Therapy: A Focused Analysis on HIV. Int J Sci: Basic Appl Res 29(3): 146-154.

3. Raymond S. (2016) The Development of New Antimicrobial Pathways: Combatting the Threat of Antimicrobial Resistance. Int J Sci: Basic Appl Res 30: 22-28.

4. Raymond S. (2018) Site Attachment Inhibition Therapeutics: Dealing with
Association and Causation Issues. Proceedings of Global Summit on Immunology and Cell Biology \& Global Congress on Bacteriology and Infectious Diseases. Amsterdam, Netherlands, June 25-26 2018.

5. Raymond S. (2016) The Role of Infectious Disease and Inflammation in Psychiatric Illness. Imp J Interdis Res 3(1): 510-513.

6. Raymond S. (2017) Site Attachment Inhibition Therapeutics: A Core Summary. J AIDS Clin Res 8: 2

7. Raymond S. (2017) Annual Conference on Microbial Pathogenesis, Infectious Disease, Antimicrobials and Drug Resistance. Toronto, Canada, August 23-24 2017; Arch Clin Microbiol 8:5.

8. Raymond S. (2018) Site Attachment Inhibition and the Application of Quantum Physics to Medicine and Surgery. J Human Soc Sci (IOSR-JHSS) 23(1): 8-12.

9. Raymond S. (2016) Combatting the global threat of antimicrobial resistance and antiviral deficiencies. Imp J Interdis Res 2(12): 676-680.

10. NIH (2018) What are genome editing and CRISPR-Cas9? Genetic home reference, U.S National Library of Medicine. Available from https://ghr.nlm.nih. gov/primer/genomicresearch/genomeediting

11. Could your computer SUE you? Machines will develop consciousness and may need 'human rights', claims Oxford professor (2017) Daily Mail. Available from https://bigthink.com/going-mental/can-computers-be-conscious

12. Computers could develop consciousness and may need 'human' rights, says Oxford professor (2016) The Telegraph, News, Science. 